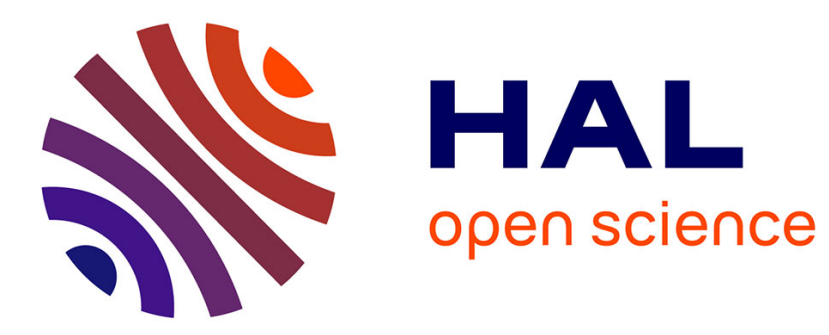

\title{
Orientation of Hydrogenic Levels by Stark Effect and sp Coherence Resulting from Direct Excitation or Molecular Dissociation
}

\author{
Maurice Lombardi, Marc Giroud
}

\section{- To cite this version:}

Maurice Lombardi, Marc Giroud. Orientation of Hydrogenic Levels by Stark Effect and sp Coherence Resulting from Direct Excitation or Molecular Dissociation. Physical Review Letters, 1976, 36, pp.409412. 10.1103/PHYSREVLETT.36.409 . hal-00974327

\section{HAL Id: hal-00974327 https://hal.science/hal-00974327}

Submitted on 6 Apr 2014

HAL is a multi-disciplinary open access archive for the deposit and dissemination of scientific research documents, whether they are published or not. The documents may come from teaching and research institutions in France or abroad, or from public or private research centers.
L'archive ouverte pluridisciplinaire HAL, est destinée au dépôt et à la diffusion de documents scientifiques de niveau recherche, publiés ou non, émanant des établissements d'enseignement et de recherche français ou étrangers, des laboratoires publics ou privés. 


\title{
Orientation of Hydrogenic Levels by Stark Effect and sp Coherence Resulting from Direct Excitation or Molecular Dissociation
}

\author{
Maurice Lombardi and Marc Giroud \\ Laboratoire de Spectrométrie Physique, Université Scientifique et Médicale de Grenoble, \\ 38041 Grenoble Cedex, France \\ (Received 4 November 1975)
}

\begin{abstract}
We have measured the predicted orientation induced by first-order Stark effect on $s p$ coherent levels of $\mathrm{He}^{+}$and $\mathrm{H}$ excited by a $100-\mathrm{keV} \mathrm{Na}^{+}$beam impinging on a cell filled with $\mathrm{He}$ or $\mathrm{H}_{2}$. We discuss the mechanism of production of $s p$ coherence by dissociative excitation of $\mathrm{H}_{2}$.
\end{abstract}

Coherence between $2 s$ and $2 p \mathrm{H}$ levels produced by passing a beam of $\mathrm{H}^{+}$through a thin carbon foil has been measured by two groups ${ }^{1}$ which used a method proposed by $\mathrm{Eck}^{2}$ : The difference of intensity between light emitted with an electric field parallel and antiparallel to the beam is proportional to coherence between even- and oddparity levels. A similar experiment has been made on Balmer- $\alpha$ light emitted by a beam of $H$ atoms excited by an electron beam and has been compared to the predictions of the Born approximation. ${ }^{3}$ More recently Macek and the present author $\mathrm{s}^{4}$ have studied the influence of even-odd coherence on the polarization of the light emitted by a hydrogenic atom excited by a foil or by collision with an ion or electron beam. The most striking result of this study is the prediction that orientation (measured by emission of circular polarization) of hydrogenic levels must appear in the $O y$ direction when an homogeneous electric field $\overrightarrow{\mathrm{E}}$, directed along $O x$, is perpendicular to the beam, directed along $O z$ [Fig. 1(a)]. This orientation changes sign with $\overrightarrow{\mathrm{E}}$. When one has no coherence between opposite-parity levels, it has been shown earlier ${ }^{5}$ that orientation can occur only if the electric field is not perpendicular (nor parallel) to the beam, and that it does not depend on the sign of $\vec{E}$ because it is due to evenorder Stark effect. The physical explanation of the coherence effect is the following. Immediately after excitation, the electron cloud is not symmetrical around the nucleus, so that there is a net value of the dipole moment of the atom which corresponds to a coherence between oppositeparity levels of the atom. In usual cases, the Coulomb attraction between nucleus and electron produces a very rapid oscillation of this coherence during the lifetime, at a frequency given by the difference in energy between opposite-parity levels, so that it is averaged out. In the hydrogenic case, due to $l$ near degeneracy, the oscilla- tion is very slow. The electric field $\vec{E}$ then rotates the electron cloud around the direction $O y$ and creates a nonzero value of the angular momemtum along $O y$, i.e., $O y$ orientation.

We have used a beam-gas instead of a beamfoil setup, for experimental convenience. This has enabled us to study for the first time evenodd coherence of $\mathrm{H}$ atoms resulting from dissociation of $\mathrm{H}_{2}$. A $20-\mu \mathrm{A}, 50-150-\mathrm{keV}$ beam of $\mathrm{Na}^{+}$ or $\mathrm{Li}^{+}$excites a vapor of $\mathrm{He}$ or $\mathrm{H}_{2}$ at a pressure of roughly $10^{-2}$ Tor $r$, which has been checked to be low enough to avoid perturbations by second collisions of the excited atom against unexcited atoms or molecules. The circular polarization of the line, selected by an interference filter, was measured by a rotating quarter-wave plate followed by a fixed Polaroid sheet. ${ }^{6}$ The linear polarization was measured by a rotating Polaroid. The modulated polarization signal was detected by a lock-in detector and stored on a multichannel analyzer for 16 to $64100-\mathrm{sec}$ sweeps. The electric field was swept synchronously with the

(a)

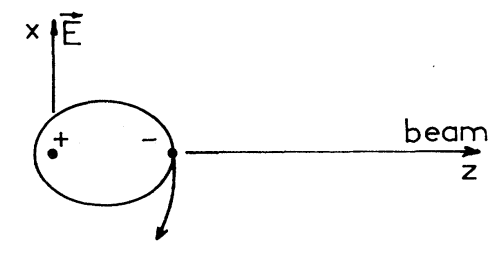

(b)

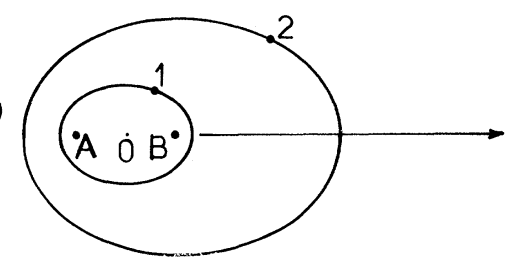

FIG. 1. Sketch of the position of various particles immediately after excitation in (a) the atomic case and (b) the molecular case if the internuclear axis happens to be in the beam direction. 


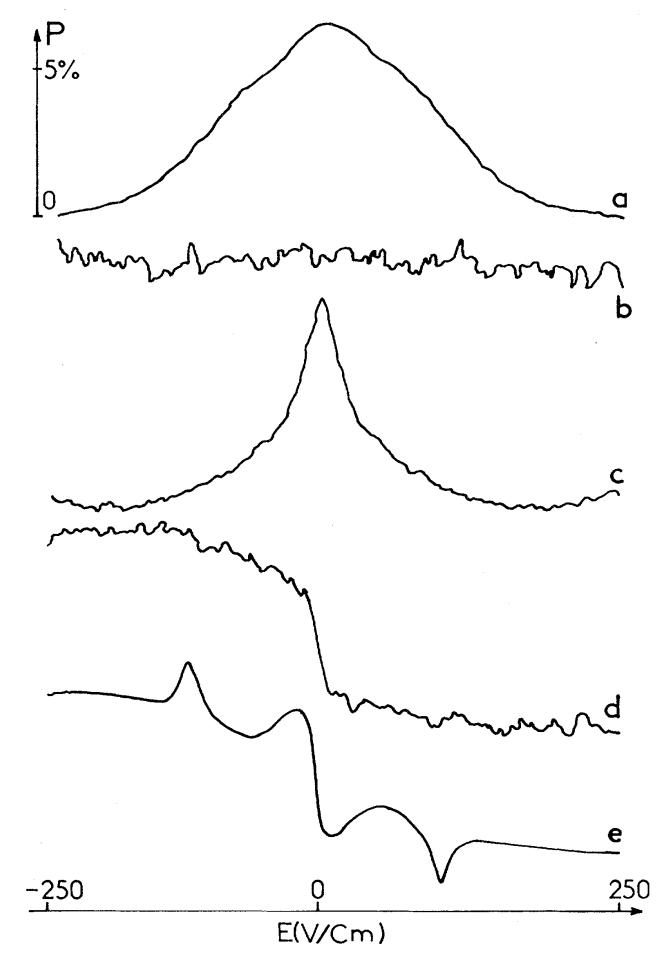

FIG. 2. Degree of linear $\left[P=\left(I_{\|}-I_{\perp}\right) /\left(I_{\|}+I_{\perp}\right)\right]$ or circular $\left[P=\left(I \sigma_{+}-I \sigma_{-}\right) /\left(I \sigma_{+}+I \sigma_{-}\right)\right]$polarization of the light emitted in the $O y$ direction. $\mathrm{Na}^{+}(110 \mathrm{keV}) \rightarrow \mathrm{He} \mathrm{I}$ $(4922 \AA)$ : trace $a$, linear; trace $b$, circular $(\times 16)$. $\mathrm{Na}^{+}(110 \mathrm{keV}) \rightarrow \mathrm{He}$ II $(4686 \AA):$ trace $c$, linear; trace $d$, circular $(\times 4) . \mathrm{Na}^{+}(70 \mathrm{keV}) \rightarrow \mathrm{H}($ Balmer $\alpha)$ : trace $e$, circular $(\times 4)$.

multichannel analyzer. The earth's magnetic field was compensated by Helmholtz coils.

Figure 2 gives some experimental curves. For He the results for the hydrogenoid He II $4686-\AA$ line are compared to those of the nonhydrogenoid He I 4922- $\AA$ line to demonstrate that circular polarization curves, due to noncoherent excitation, are similar.

We have done similar experiments on hydrogen atoms resulting from the dissociation of $\mathrm{H}_{2}$ (Fig. 2 , trace $e$ ). In the Balmer- $\alpha$ case, even-odd coherence depend on the three elements $\rho\left(s_{0}, p_{0}\right)$, $\rho\left(p_{0}, d_{0}\right)$, and $\rho\left(p_{1}, d_{1}\right)$ of the density matrix. ${ }^{3}$ Qualitatively, the possibility of extracting these parameters from the experimental data is based on the following remarks. First, at low field, when one can neglect the mixing of different $j$ levels, the circular-polarization signal is the sum, weighted by the searched coherence parameters, of several dispersion curves of widely different widths corresponding to $s_{1 / 2} p_{1 / 2},\left|m_{J}\right|=\frac{1}{2}$, and $p_{3 / 2} d_{3 / 2},\left|m_{J}\right|=\frac{1}{2}, \frac{3}{2}$ electric-field mixings. Sec-
TABLE I. Experimental coherence parameters. $\mathrm{Na}^{+}(70 \mathrm{keV}) \rightarrow \mathrm{H}_{\alpha}$ [relative units: The imaginary part of $\rho\left(s_{0}, p_{0}\right)$ has been normalized to 1$]$. The error bars are 1 standard deviation. They do not include the systematic uncertainty due to a small cascade effect.

$$
\begin{array}{lr}
\rho\left(s_{0}, p_{0}\right)= & -0.180 \pm 0.031+i(-1 \pm 0.016) \\
\rho\left(p_{0}, d_{0}\right)= & 0.055 \pm 0.016+i(-0.617 \pm 0.016) \\
\rho\left(p_{1}, d_{1}\right)= & 0.070 \pm 0.016+i(-0.445 \pm 0.008)
\end{array}
$$

ond, the discrimination between the real and the imaginary parts of the $\rho$ components is based on the shape of the crossing at $105 \mathrm{~V} / \mathrm{cm}$. A detailed analysis ${ }^{7}$ shows that this crossing has an absorption shape for imaginary $\rho$ and a dispersion shape for real $\rho$. Since this would not be enough to extract unambiguously all the parameters, we have increased the experimental information in the following ways. First we have measured, on the light emitted along $O y$, the difference between linear polarization at $+45^{\circ}$ and $-45^{\circ}$ to $\mathrm{Oz}$. Second we have made the same measurements (linear and circular polarization as a function of electric field) in the presence of a magnetic field along $O z$. All these measurements can be shown to depend only, but in different ways, on the searched coherence parameters. ${ }^{4,7}$ Finally, we have made a global fit of all the available experimental curves with computer-calculated theoretical line shapes (taking into account the hyperfine structure) with the coherence parameters (and unphysical quantities such as line bases) as adjustable parameters. The resulting fit was good, but there remained a slight discrepancy, probably due to cascade effects. The results are given in Table I.

We shall now discuss the origin of even-odd coherence in atoms resulting from molecular dissociation, since this is a less obvious phenomenon than in the direct-excitation case, and since this can give information on the dissociation process. It is known that the dissociated atoms are linearly polarized, ${ }^{8}$ and the mechanism of production of this linear polarization has been discussed by Van Brunt and Zare. ${ }^{9}$ It is then tempting to try to extend the argument of Van Brunt and Zare to explain our results. It was substantially as follows. The molecule is first excited and perhaps ionized to a molecular level which then dissociates or predissociates. If the dissociation occurs through a well-defined molecular level and with a well-defined interatomic direction, the resulting atoms are usually in a 
sharp quantum state with respect to the direction of dissociation and this leads to a high degree of polarization along this direction. One has then to average over the various directions of dissociation. If there is equal probability of dissociation in any direction, the polarization is averaged out. The experimental existence of polarization is then evidence of an anisotropy of the dissociation direction. Various theoretical and direct experimental studies have been made of the anisotropy of the fragments resulting from dissociative excitation or ionization of $\mathrm{H}_{2}$ and $\mathrm{H}_{2}{ }^{+} \cdot{ }^{10}$

The large-internuclear-distance $(R)$ form of the symmetrized electronic wave function corresponding to the dissociation of a pure $\mathrm{H}_{2}$ molecular level into an unexcited $\mathrm{H}$ atom and an atom excited in the $n=2, m_{L}=0$ state is, with usual notations of molecular orbitals in the separatedatom limit, and with parallel frames of reference on $A$ and $B$ [Fig. 1(b)],

$$
\begin{aligned}
& \varphi_{\mathrm{u}, \mathrm{g}}=\psi+(-)^{\mathrm{g} I} \psi, \\
& \psi=\mathcal{Q}\left\{\psi_{\sigma 1_{S B}}(1)\left[a \psi_{\sigma 2 s A}(2)+b \psi_{\sigma 2 D A}(2)\right]\right\}, \\
& I \psi=\mathbb{Q}\left\{\psi_{\sigma 1 s A}(1)\left[a \psi_{\sigma 2 s B}(2)-b \psi_{\sigma 2 D B}(2)\right]\right\},
\end{aligned}
$$

where $\mathcal{Q}$ is the antisymmetrizer of the two electrons, $(-)^{\mathrm{g}}=+1$ for gerade and -1 for ungerade levels, and $I$ is the inversion on the center $O$ of the molecule. If the unexcited $\mathrm{H}$ atom is replaced by $\mathrm{a} \mathrm{H}^{+}$ion (we have no experimental indication that our even-odd coherence comes from dissociation rather than dissociative ionization), $a$ $= \pm b=1 / \sqrt{2}$ and this form of $\psi$ is due to mixing of the degenerate $s, 0$ and $p, 0$ levels by the electric field of the ion. In the neutral case, an effective mixed form of $\psi$ at the end of dissociation is a probable consequence of the variation of the character of the wave function with $R^{11}$ and of the posibility of diabatic transitions between these neardegenerate levels.

The important point is the change of sign before $b$, which is due to the oddness of the $p$ or bital under inversion. The $\psi$ and $I \psi$ components of $\varphi$, which have equal probability in pure gerade or ungerade wave functions, and which correspond to backward and forward dissociations, give opposite values $\pm a b^{*}$ to the coherence $\rho(s, p)$. The observation of a nonzero value of $\rho(s, p)$ is then evidence that the intermediate molecular state is not a pure molecular $\mathrm{u}$ or $\mathrm{g}$ state. It can, however, be a coherent mixing $\alpha\left(\varphi_{\mathrm{u}}+\beta \varphi_{\mathrm{g}}\right)$ of molecular wave functions, since this gives different weights, $\alpha(1+\beta)$ and $\alpha(1-\beta)$, to the $\psi$ and $I \psi$ components. This is possible, since $\psi$ corre- sponds to an electronic excited cloud centered on $A$ and $I \psi$ on $B$ [Fig。 1(b)] and since the primary excitation process is likely to produce a difference between them. This coherence $\beta$ between $\varphi_{\mathrm{u}}$ and $\varphi_{\mathrm{g}}$ must also survive the molecular dissociation. This implies that the $u$ and $g$ curves involved (which can be other than the adiabatic ones) follow each other closely enough so that the oscillation of $\beta$ at a frequency given by their difference in energy does not average it out.

This interpretation implies also that there must be a forward-backward asymmetry in the dissociation. However this is not strictly true if one takes into account the fact that other molecular states can be involved. In particular, the orthogonal state with $a, b$ in $\psi$ replaced by $b,-a$ could compensate for the anisotropy and add for $s p$ coherence. This is not a completely unreasonable hypothesis since the resulting molecular wave function corresponds to an outer electron excited in a Rydberg sp coherent orbital (i.e., not centered on $O$ ) and the inner electron in an isotropic repulsive one. In this case, $s p$ coherence of the dissociation products is not due to an asymmetry of the direction of dissociation but to the anisotropic character of the outer orbital. The same problem exists for the Van Brunt and Zare interpretation of polarization. This second model is certainly not valid at threshold energy with electron excitation, since the four molecular orbitals, which this initial wave function decomposes into, have very different unitedatom limits, ${ }^{11}$ but it cannot be ruled out at once for ion excitation with a great excess of energy. For the interpretation of $s p$ coherence, it would then be very interesting to measure directly the forward-backward asymmetry of the dissociation products. Such an asymmetry has indeed been o'sserved with electron excitation ${ }^{10}$ but has been attributed to the recoil of the molecule under primary excitation. ${ }^{12}$

These experiments have been done at the Laboratoire de Spectrométrie Ionique et Moléculaire of Lyon, directed by Professor M. Dufay, with equipment built by M. Carré. Assistance by G. Do Cao and A. Zgainsky in running the accelerator and taking the data is gratefully acknowledged.

${ }^{1}$ I. A. Sellin, J. R. Mowat, R. S. Peterson, P. M. Griffin, R. Laubert, and H. H. Haselton, Phys. Rev. Lett. 31, 1335 (1973); A. Gaupp, J. Andrä, and J. Macek, 
Phys. Rev. Lett. 32,6 (1974).

${ }^{2}$ T. G. Eck, Phys. Rev. Lett. 31, 270 (1973).

${ }^{3}$ H. Mahan, R. V. Krotkov, A. C. Gallagher, and S. J. Smith, Bull. Am. Phys. Soc. 18, 1506 (1973); R. Krotkov, Phys. Rev. A 12, 1793 (1975).

${ }^{4}$ M. Lombardi, M. Giroud, and J. Macek, Phys. Rev. A 11, 1114 (1975).

${ }^{5} \mathrm{M}$. Lombardi and M. Giroud, C. R. Acad. Sci. 266, 60 (1968); M. Lombardi, J. Phys. (Paris) 30, 631 (1969).

${ }^{6}$ M. Pavlovic and F. Laloë, J. Phys. (Paris) $\underline{31}, 173$ (1970).

${ }^{7}$ M. Giroud, M. Lombardi, and M. Glass, to be published.

${ }^{8}$ D. A. Vroom and F. J. De Heer, J. Chem. Phys. 50, 580 (1969).

${ }^{9}$ R. J. Van Brunt and R. N. Zare, J. Chem. Phys. $\underline{48}$,
4304 (1968).
${ }^{10}$ G. H. Dunn, Phys. Rev. Lett. $\underline{8}, 62$ (1962); R. J. Van Brunt, J. Chem. Phys. 60, 3064 (1974); R. N. Zare, J. Chem. Phys. 47, 204 (1967); T. F. O'Malley and H. S. Taylor, Phys. Rev. 176, 207 (1968); R. J. Van Brunt and L. J. Kieffer, Phys. Rev. A 2 , 1293 (1970); M. Misakian and J. C. Zorn, Phys. Rev. A $\underline{6}, 2180$ (1972); A. Crowe and J. W. McConkey, J. Phys. B $\underline{6}$, 2088 (1973).
${ }^{11}$ T. E. Sharp, At. Data 2,119 (1971).
${ }^{12}$ R. J. Van Brunt and L. J. Kieffer [Phys. Rev. A $\underline{2}$, 1899 (1970)] argued that a center-of-mass, forward- backward asymmetry could come through excitation of a mixed $\Pi_{u} \Delta_{u}$ molecular state. However their final for- mula is certainly in error since it destroys the invari- ance of probability of dissociation by rotation around the electron-beam axis.

\title{
Lattice Softening and Anisotropy at ${ }^{119} \mathrm{Sn}$ Sites in $\mathrm{SnMo}_{6} \mathrm{~S}_{8} \dagger$
}

\author{
C. W. Kimball, L. Weber, and G. Van Landuyt \\ Northern Ilinois University, DeKalb, Mlinois 60115 \\ and \\ F. Y. Fradin, B. D. Dunlap, and G. K. Shenoy \\ Argonne National Laboratory, Argonne, Ilinois 60439 \\ (Received 26 November 1975)
}

\begin{abstract}
The vibrational motion of $\mathrm{Sn}$ in the high-critical-field superconductor $\mathrm{SnMo}_{6} \mathrm{~S}_{8}$ has been studied with the ${ }^{119} \mathrm{Sn}$ Mössbauer effect. The Mössbauer thermal shift and recoil-free fraction are found to contain large anharmonic contributions from the soft modes. The soft vibrational motion contributes substantially to the mass enhancement $\lambda$.
\end{abstract}

There is considerable current interest in the ternary superconductors based on the molybdenum sulfides. Recent studies indicate superconducting transition temperatures $T_{c}$ as high as $14.4 \mathrm{~K}$ and upper critical fields as high as 600 $\mathrm{kG}^{1}$ The third element in the ternaries is clearly of great importance in determining the superconducting properties of these compounds. For example, when the third element is removed by chemical treatment, a slight triclinic deformation takes place and no superconducting transition is detected to less than $1 \mathrm{~K}^{2}$ Thus, investigations of the local properties of this component are of importance in understanding these materials. In this regard, $\mathrm{SnMo}_{6} \mathrm{~S}_{8}$ provides an interesting case to study. While many of these materials show lattice instabilities with a first-order phase transition, neither crystallographic and resistivity ${ }^{3}$ nor heat-capacity and susceptibility ${ }^{4}$ measurements show a transition for the Sn-based compounds. In addition, this class of compounds has the largest pressure dependence of $T_{c}$ yet found for any superconductor. ${ }^{5}$ In this Letter we report a Mössbauer study of the lattice properties of ${ }^{119} \mathrm{Sn}$ in $\mathrm{SnMo}_{6} \mathrm{~S}_{8}$. The measurements show (1) anisotropic mean-square lattice displacements with $\left\langle z^{2}\right\rangle-\left\langle x^{2}\right\rangle<0$ and a strong deviation from harmonic behavior; (2) a temperature-dependent line shift indicative of large anharmonicity and soft modes; (3) a possibly large contribution of the soft vibrational modes to the mass enhancement $\lambda$, and hence to the high $T_{c}$.

Mössbauer spectra were obtained as a function of temperature and external magnetic field on a $\mathrm{SnMo}_{6} \mathrm{~S}_{8}$ sample having $T_{c}=10.8 \mathrm{~K}$. X-ray measurements showed a small quantity of $\alpha$-Mo and the Mössbauer spectra showed $\sim 5 \% \beta$-Sn present in addition to the rhombohedral ( $\mathrm{Mo}_{6} \mathrm{Se}_{8}$-type) $\mathrm{SnMo}_{6} \mathrm{~S}_{8}$. The $\beta$-Sn spectra were stripped from the data using the results of Rothberg, Grumard, and Benczer-Koller ${ }^{6}$ and Hohenemser. ${ }^{7}$ The linewidth of the absorber versus a $15-\mathrm{mCiV}(\mathrm{Sn})$ 